\title{
TRATAMENTO DA CRIPTOCOCOSE DO SISTEMA NERVOSO PELO AMPHOTERICIN B
}

\author{
DANTE GIORgI * \\ Paulo Pinto Pupo**
}

\author{
JoÃo Baptista dos Reis * \\ José Geraldo de Camargo Lima***
}

O presente trabalho tem por finalidade registrar resultados que reputamos os mais promissores na evolução da terapêutica da criptococose humana, obtidos com o emprêgo do Amphotericin B.

Sendo esta afecção moléstia grave, com incidência não rara na literatura médica, com incidência entre nós já comprovada por vários autores (Almeida e Lacaz ${ }^{2}$; Almeida, Lacaz e Monteiro Sales ${ }^{3}$; Cortez ${ }^{16}$; Clausell ${ }^{12}$; Duarte ${ }^{24}$; Fialho ${ }^{26}$; Longo e col.101; Reis e Bei ${ }^{18}$; Amorim e Pasquallucci ${ }^{4}$; Tolosa, Spina-França e Lacaz ${ }^{93}$; Benício ${ }^{102}$ ), torna-se evidente o interêsse do registro de caso de cura com o medicamento novo.

$\mathrm{Na}$ evolução dos conhecimentos sôbre a criptococose do sistema nervoso central duas fases bem distintas se nos apresentam.

$\mathrm{Na}$ primeira, o problema era o do diagnóstico em vida. Conhecida já há 54 anos, pois foi descrita inicialmente como afecção do sistema nervoso central por von Hansemann em 1905, à qual seguiram-se as descrições de Turk em 1907 e de Stodart e Cutler em 1916, esta afecção constituiu achado de necropsia na maioria dos casos desde então relatados. Em vida o seu diagnóstico foi confundido com o de outras infecções e parasitoses do sistema nervoso central, permanecendo ignorada a verdadeira etiologia dos casos.

Foram os dados fornecidos pelo exame do líqüido cefalorraqueano (LCR) que vieram permitir o diagnóstico com segurança. Ele depende da demonstração do fungo já que as demais alterações dêsse líqüido não são suficientes para o diagnóstico diferencial entre a meningite por êle provocada e as causadas pela tuberculose, por certos abscessos ou por outras encefalites. Entre nós, Reis e Bei 78 , contribuiram demonstrando a possibilidade dêsse diagnóstico pelo emprêgo sistemático do método de pesquisa do fundo pela tinta da China e da cultura, em todos os casos em que tal suspeita diagnóstica esteja em mente. Com tal prática se tornou possivel o diagnóstico na maioria dos casos.

Trabalho do Serviço de Neurologia da Escola Paulista de Medicina (Prof. Paulino W. Longo), apresentado no Departamento de Neuro-Psiquiatria da Associação Paulista de Medicina em 5 maio 1959. *Assistente; ** Chefe de Clinica; *;* Médico interno. 
Tem havido realmente aumento na publicação de casos de criptococose humana na literatura mundial, aumento êsse que vem sendo motivo de controvérsias. Assim, autores como Rubin e Furcolow 82 acreditam na possibilidade de um aumento real da infestação pelo criptococo, aumento êste causado particularmente pelo abuso dos antibióticos de largo espectro e pelo emprêgo generalizado da terapêutica pelos esteróides. Nós não acreditamos nisso. A nosso ver êsse aumento do número de casos de criptococose se deve ao aperfeiçoamento dos métodos diagnósticos.

Em nosso Serviço, onde se emprega tais métodos rotineiramente desde 1953, a incidência de torulose não aumentou em 6 anos. Não houve, pois, pelo menos em nosso meio, aumento real da incidência da afecção. Os dados projetados no quadro 1 disso são documento.

\begin{tabular}{|c|c|c|c|c|c|c|}
\hline A no & 1953 & 1954 & 1955 & 1956 & 1957 & 1958 \\
\hline No de casos & 2 & 1 & 2 & 5 & 2 & 2 \\
\hline
\end{tabular}

Quadro 1 - Casos de criptococose do sistema nervoso registrados no Serviço de Neurologia da Escola Paulista de Medicina.

Vencido o problema do diagnóstico, restou o da terapêutica desta meningoencefalite, anteriormente quase sempre mortal. Se, quanto ao diagnóstico houve grande progresso, o mesmo não se pode dizer quanto à terapêutica. Esta, tinha por base predominantemente os iodetos 11, 17, 27, 30, 31, 38, $50,53,61,65,67,68,75,76,77,80,85,90,96,99$, e as sulfas $6,11,12,13,17,27,30,31$, $32,35,38,40,50,53,61,65,71,74,75,76,77,79,80,83,92,93,96$ 99. Esses medicamentos, que se mostraram ativos em outras micoses, fracassaram, entretanto, na grande maioria dos casos de criptococose do sistema nervoso central, confirmando as experiências sem sucesso in vitro. Outras drogas foram experimentadas, igualmente sem qualquer resultado, tanto in vitro como in vivo. Assim a penicilina ${ }^{19}, 27,53,54,87$, a sulfonamida ${ }^{19}$, a actidione ${ }^{7}, 25,27,39,43,46,90,99$, a tirotricina, estreptomicina, tirocidina, subtilin, tirotricin, gramicidin, bacitracin e os ácidos gordurosos ${ }^{54}$, a aureomicina, cloromicetina e terramicina ${ }^{27}$, a prata coloidal ${ }^{8 \overline{5}}$. Outras drogas, que haviam dado bons resultados experimentalmente como o bacilomicin ${ }^{48}$, protoanemonin ${ }^{49}$ e streptotricin 81 não corresponderam quando usadas no homem ${ }^{9,10}$. O mesmo se deu com a hipertermia ${ }^{8}$, apesar dos ótimos resultados experimentais ${ }^{54,5 \pi, 56,81}$ e com a hialuronidase ${ }^{23}$. Também o gotejamento de álcool a $5 \%$ na veia não surtiu efeito 54,68 .

Da revisão da literatura encontram-se apenas 2 casos tidos como curados. O primeiro é o de Marshall e Teed ${ }^{65}$, tratado com sulfadiazina e iodeto de potássio, e que estava assintomático já há 6 anos. O segundo, de Levin e Roux (cit. por Mosberg e Arnold i1), foi tratado com penicilina, sulfapiridina e iodeto de potássio, com um seguimento satisfatório de 11 meses. Em ambos houve normalização do LCR. Nos demais casos regis- 
trados na literatura a medicação empregada não surtiu efeito algum 2, 6, 7, 9, $11,12,13,14,17,25,27,30,31,32,33,35,38,39,40,42,43,46,50,53,54,60,64,67,68,69$, $70,71,73,74,75,76,77,79,80,83,85,89,90,92,93,96,97,99,100$.

No gráfico 1 analisamos os casos da literatura acima citados, em número de 51, nos quais havia referencia do tempo de sobrevida do paciente. Assim verificamos que a evolução se fêz em um tempo médio de 3 a 6 meses, confirmando as idéias de Littmann e Zimmermann (cit. por Rubin e Furcolow ${ }^{82}$ ), evolução essa semelhante à daqueles casos não tratados, segundo os informes da literatura 1, 19, 24, 3i, 44, 59,67, $72,76,86,90,96,98$.

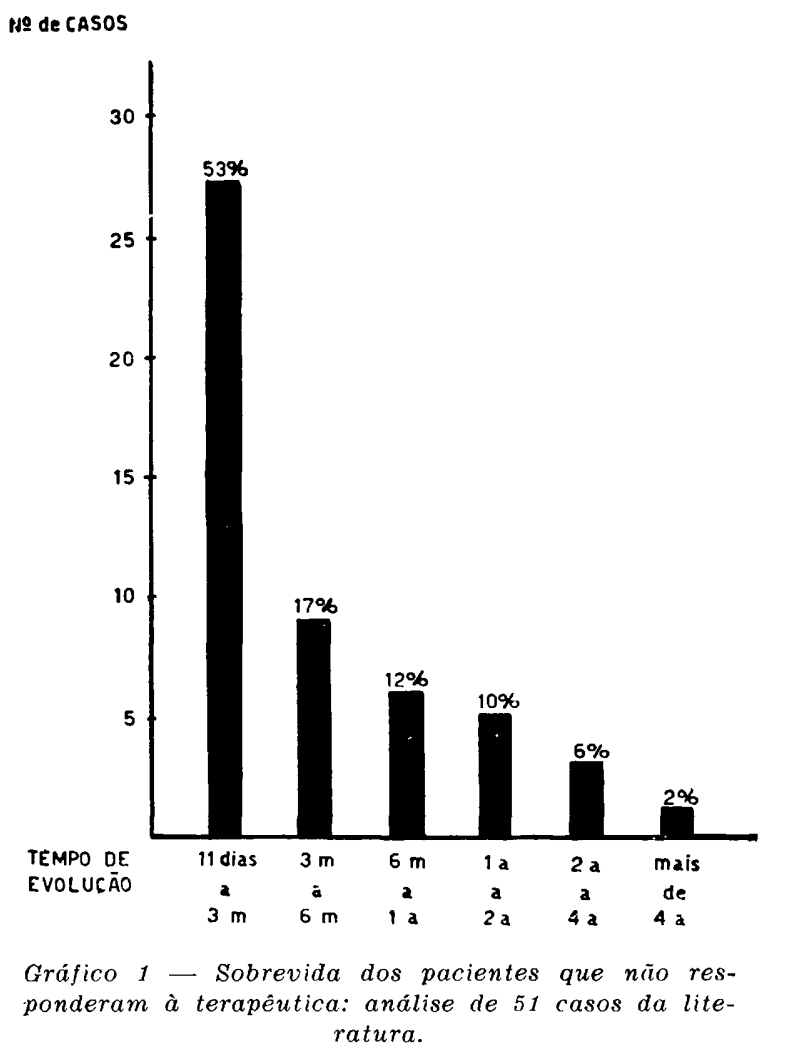

Em síntese, tudo o que se viu foi tôdas as terapêuticas até então tentadas se mostrarem ineficazes no combate à criptococose e esta evoluir de maneira fatal dentro de 6 meses na grande maioria dos casos, e, em poucos dêles, evoluir de modo crônico, com remissões e recidivas, sob a forma de uma meningoencefalite tórpida. O diagnóstico perfeitamente possível, mas as possibilidades terapêuticas pràticamente nulas.

Entretanto, a partir de 1955, êsse panorama tende a se modificar com o aparecimento de um novo antibiótico de poder fungicida evidente, o Ampho- 
tericin $\mathrm{B}$, derivado de uma raça de streptomyces; sua ação fungicida foi demonstrada em vários trabalhos experimentais $29,34,41,45,58,66,88$.

$\mathrm{Na}$ literatura encontramos referências de 27 casos de criptococose do sistema nervoso central tratados com o Amphotericin $\mathrm{B} 5,18,20,21,22,28,47,51$, $52,62,82,91,94,95 *$ e os resultados obtidos com essa nova droga, se comparados com os obtidos anteriormente pelos outros meios terapêuticos, são encorajadores. Assim, entre êsses 27 casos, apenas 7 evoluíram ràpidamente para a morte. Dos 20 restantes, 6 ficaram completamente curados (contrôle clínico e do LCR), outros 6 apresentaram melhoras clínicas acentuadas, porém com LCR ainda alterado na citologia e na taxa de proteínas, embora fôsse negativa a cultura. Em 6 casos que haviam apresentado no início melhoras clínicas sem normalização do LCR, houve recidiva. Os dois casos restantes poucas melhoras apresentaram. Não nos foi possível com êsse material organizar uma curva de sobrevida semelhante à do gráfico 1 , pois o tempo de observação com esta nova terapêutica é ainda demasiado curto.

O Amphotericin $\mathrm{B}^{* *}$ é empregado na dose de $1 \mathrm{mg} / \mathrm{kg}$ de pêso, dissolvido em $500 \mathrm{ml}$ de sôro glicosado a $5 \%$ e administrado gòta-a-gôta na veia, em dias alternados ou diàriamente. A dose total por série não deve ultrapassar de $1,0 \mathrm{~g}$. Por via raqueana a dose é de 0,5 a $1 \mathrm{mg}$ dissolvido em 10 $\mathrm{ml}$ de água bidestilada.

No homem êsse antibiótico provoca náuseas, vômitos, calafrios, dôres pelo corpo e, mais raramente, determina flebites. Sua ação tóxica mais grave se exerce sôbre o rim, determinando retenção de uréia e obrigando a suspensão do medicamento por uns dias. Os efeitos secundários do Amphotericin B são, entretanto, evitados ou debelados com o emprêgo de anti-histaminicos, novalgina, ou corticosteróides.

Como vimos no quadro 1 , o nosso material clínico se compõe de 14 casos, todos diagnosticados em vida, pela demonstração do Criptococcus neoformans no LCR (pelo método da tinta da China e pela cultura). Êles foram evidenciados no período de 1953 a 1958, quando a pesquisa pela tinta da China se tornou rotina. As terapêticas de que lançamos mão foram as mais diversas, tais como penicilina, sulfas, actidione, iodetos e terramicina, sem contudo obter resultado favorável. Os dois casos mais recentes foram tratados com o Amphotericin $B$ e são êsses os que relatamos no presente trabalho.

\section{OBSERVAÇÕES}

CASo 1 - I.J., branca, brasileira, internada em 15 de setembro de 1957. Queixa: cefaléia intensa acompanhada de vômitos, iniciada há um ano. Exame geral - Paciente afebril, apática, com raquialgia e rigidez de nuca; edema bilateral das papilas ópticas. O exame do LCR demonstrou uma meningite linfocitária com presença de numerosos Criptococcus neoformans.

* Êste trabalho já estava elaborado quando chegou às nossas mãos o de Smith, G. W. - Treatment of torula encephalitis with amphotericin B. J. Neurosurg., 15: 572-575, 1958.

** Agradecemos a Squibb \& Sons, Produtos Farmacéuticos o fornecimento do medicamento. 
Como naquela ocasião ainda não dispuséssemos de Amphotericin B, o tratamento feito visou principalmente combater a hipertensāo intracraniana com drenagem continua do LCR (por meio de dreno de polietileno de demora na raque), Diamox e sôro glicosado hipertônico endovenoso. Como tentativa de tratamento curativo administramos por via oral ácido undecilènico. Com êsse tratamento a paciente apresentou uma melhora inicial; porém em fevereiro de 1958, em pleno tratamento, apresentou nova exacerbação da sintomatologia. O exame do LCR, feito nessa ocasião, demonstrou 18 criptococos por $\mathrm{mm}^{3}$.

Foram administradas, a partir desta data, 4 séries de 10 injeções de Amphotericin B (50 mg por injeção), em dose total de $500 \mathrm{mg}$ por série, não havendo reação colateral de importância.

Pelas modificações do LCR observadas no decurso do tratamento, podemos avaliar a evoluçāo mais favorável da moléstia.

\begin{tabular}{|c|c|c|c|c|c|c|}
\hline D a t a & Aspecto & $\begin{array}{c}\text { Citol. por } \\
\mathrm{mm}^{3}\end{array}$ & $\begin{array}{l}\text { Prot. } \\
\text { mg\% }\end{array}$ & $\begin{array}{l}\text { Glic. } \\
\text { mg\% }\end{array}$ & $\begin{array}{l}\text { Criptoco- } \\
\text { cos por } \\
\mathrm{mm}^{3}\end{array}$ & Terapêtica \\
\hline $15-9-57$ & Opal. & 117,0 & 68 & 57 & 3,2 & \\
\hline $21-1-58$ & Limp. & $\mathbf{5 3 , 0}$ & 52 & 42 & 2,5 & \\
\hline $14-2-58$ & Limp. & 38,0 & 54 & 32 & 18,0 & Início $1^{\text {a }}$ série \\
\hline $25-3-58$ & Opal. & 12,0 & 36 & 53 & 0,2 & \\
\hline $8-5-58$ & Opal. & 41,4 & 56 & 34 & 18,8 & Início $2^{\mathrm{a}}$ série \\
\hline $28-6-58$ & Limp. & 8,0 & 32 & 52 & 1,8 & \\
\hline $15-7-58$ & Opal. & 122,4 & 58 & 44 & 21,0 & Inicio $3^{a}$ série \\
\hline $23-9-58$ & $\begin{array}{l}\text { Xant. } \\
\text { Opal. }\end{array}$ & 96,0 & 54 & 46 & 2,0 & \\
\hline $18-3-59$ & Limp. & 53,4 & 140 & 28 & 0,7 & Inicio $4^{8}$ série \\
\hline $24-4-59$ & $\begin{array}{l}\text { Opal. } \\
\text { Xant. }\end{array}$ & 115,6 & 280 & 9 & 0,1 & \\
\hline
\end{tabular}

Quadro 2-Caso 1. Quadro evolutivo do LCR antes e durante a terapêtica com Amphotericin B. Legenda: Citol. = citologia; Prot. = proteinas; Glic. = glicose; opal. = opalescente; limp. = limpido; xant. $=$ xantocrômico.

Nesta seqüência vemos que, sem chegar à normalizacão, houve sempre melhoria do quadro do LCR, quando das séries do Amphotericin B. Com exceção da última, tôdas as aplicaçôes foram iniciadas em pleno periodo de exacerbação clinica e os exames de LCR, feitos nessas ocasiões, sempre se mostraram francamente positivos. Melhora evidente, tanto clínica como do LCR, se nota no decurso das aplicaçōes.

Esta paciente está com uma sobrevida de 20 meses, bem maior, portanto, do que a sobrevida dos casos tratados antes do advento do Amphotericin B. Estamos, pois, diante de uma paciente portadora de criptococose do sistema nervoso central, que evolui já há meses de modo crônico, em cujas recidivas o Amphotericin B tem se mostrado nitidamente eficaz como meio terapêutico. 
CASo 2 - M.L.T., branca, brasileira, internada em 17 de junho de 1958 . Sua moléstia se iniciou há mais ou menos 2 meses, com cefaléia tórpida, difusa, predominantemente occipital, com surtos de acentuação; em um dêsses surtos apresentou tonturas, vômitos e febre. Antes de sua internação, havia sido submetida a tratamento por vários antibióticos, com diagnóstico de meningite, sem que tivesse sido determinada a etiologia. Exame clinico - Paciente febril $\left(38,5^{\circ} \mathrm{C}\right)$, rigidez de nuca, edema de papila bilateral e paresia do nervo motor ocular externo à direita. o exame de LCR feito nessa ocasião revelou um quadro de meningite linfocitária com presença de Criptococcus neoformans.

A partir de 21 de junho de 1958, foi instituido o tratamento pelo Amphotericin $B$ por via endovenosa em solução de sôro glicosado $5 \%$ na dose de 50 mg por vez, e administrado em dias alternados. Esta primeira série constou de 10 injeções com um total de $500 \mathrm{mg}$ de Amphotericin B. Após isso, a paciente apresentava apenas edema de papila bilateral, sem nenhuma queixa. O LCR que de inicio apresentava 15 criptococos por $\mathrm{mm}^{3}$, ao fim da primeira série de injeções apresentava apenas 0,2 criptococos por $\mathrm{mm}^{3}$. A paciente teve alta hospitalar levando prescrição de medicação tônica geral. Reexaminada em 11 de setembro de 1958, não apresentava qualquer queixa; objetivamente encontramos apenas papilas de bordos pouco nitidos. O exame do LCR nessa ocasião já se mostrou negativo para criptococos mas ainda com alteração célulo-protêica. Foi ela então submetida a uma segunda série de Amphotericin B, semelhante a anterior. No final desta a paciente se mostrava assintomática e o LCR, se bem que melhorado, ainda não era normal. A paciente teve então alta hospitalar. Reexaminada um mês após, continuava assintomática e agora com LCR absolutamente normal. Esta paciente continua sob observação passando perfeitamente bem, sendo que seu último exame data de 5 de agôsto de 1959. Durante essas duas aplicações de Amphotericin B a paciente apresentou, em certas ocasiōes, calafrios e náuseas, que desapareceram com o diminuir da velocidade de introdução do medicamento. Em apenas uma ocasião teve reação de calafrio mais violenta, que desapareceu com novalgina endovenosa. Como reação de certa importância teve, quando da aplicação da primeira série, aumento da taxa de uréia, que atingiu a $150 \mathrm{mg} \%$ no sangue; bastou suspender o tratamento por 3 dias e tudo se normalizou.

\begin{tabular}{|c|c|c|c|c|c|c|}
\hline $\mathrm{D}$ a $\mathrm{t} \mathrm{a}$ & Aspecto & $\begin{array}{l}\text { Citol. por } \\
\text { por } \mathrm{mm}^{3}\end{array}$ & $\begin{array}{l}\text { Prot. } \\
\text { mg\% }\end{array}$ & $\begin{array}{l}\text { Glic. } \\
\text { mg\% }\end{array}$ & $\begin{array}{l}\text { Criptococos } \\
\text { por } \mathrm{mm}^{3}\end{array}$ & Terapêutica \\
\hline $18-6-58$ & Limp. & 80,0 & 56 & 13 & 0,5 & \\
\hline $21-6-58$ & Limp. & 74,0 & 32 & 14 & 15,0 & Inicio $1^{\mathrm{a}}$ série \\
\hline $19-7-58$ & Opal. & 101,0 & 31 & 61 & 0,2 & \\
\hline $11-9-58$ & Limp. & 11,0 & 28 & 57 & $\begin{array}{l}\text { Neg. } \\
\text { cult. neg. }\end{array}$ & Inicio $2^{\text {a }}$ série \\
\hline $30-9-58$ & Limp. & 10,0 & 52 & 48 & Neg. & \\
\hline $19-1-59$ & Limp. & 2,4 & 18 & 52 & Neg. & \\
\hline $5-8-59$ & Limp. & 0,8 & 19 & 72 & Neg. & \\
\hline
\end{tabular}

Quadro 3 - Caso 2. Evolução das alterações do LCR. Legenda idêntica à do quadro 2.

Trata-se pois de um caso de meningite por criptococos, que cedeu por completo em três meses de tratamento; a paciente se apresenta curada clinicamente e com LCR normal, sem parasitas demonstráveis diretamente ou pela cultura. 


\section{CONCLUSAO}

Comparando os resultados satisfatórios obtidos pelo Amphotericin B nestas duas pacientes com os até então obtidos por outros meios terapêuticos, somos obrigados a acreditar que também o problema terapêutico desta afecção se encontra em vias de solução favorável. Nossos resultados e os de Utterback ${ }^{94}$, Appelbaum e Shtokalko ${ }^{5}$, Rubin e Furcolow 82 nos autorizam a tal.

\section{RESUMO}

Os autores descrevem a evolução dos conhecimentos sôbre a meningoencefalite produzida pelo Criptococcus neoformans, analisando as dificuldades anteriormente havidas para o diagnóstico em vida, as quais foram superadas depois que se usou rotineiramente a pesquisa do cogumelo no LCR pelo método de coloração de tinta da China e pela cultura. Assinalam, a seguir, o aparecimento do Amphotericin $B$, novo antibiótico fungicida que veio modificar o prognóstico desta afecção.

Apresentam sua experiência com 14 casos de meningite por criptococos diagnosticados em vida e referem pormenorizadamente 2 casos tratados com o Amphotericin B. O primeiro, de paciente portadora desta afecção, evoluindo de maneira crônica, com períodos de exacerbação que melhoram nìtidamente com o Amphotericin B e que está com uma sobrevida de 20 meses. O segundo, de paciente portadora de forma aguda desta afecção que teve cura clínica e está com LCR absolutamente normal, com seguimento de 11 meses. Em ambos os casos foi usada a via endovenosa, sendo feitas séries de 10 injeções, cada uma de $50 \mathrm{mg}$ de Amphotericin B em $500 \mathrm{ml}$ de sôro glicosado a $5 \%$. No primeiro caso foram feitas 4 séries e no segundo apenas duas. Não foram registradas reações colaterais de importância.

Os autores terminam considerando o Amphotericin B como um elemento que veio tornar muito mais favorável o prognóstico desta afecção até então mortal.

\section{SUMMARY}

The treatment of neuro-criptococcosis with the Amphotericin $B$.

The authors made a review of the diagnostic difficulties in meningoencephalitis by the Criptococcus neoformans, in vivo, today easier by special methods such as China ink and culture in the CSF.

From their diagnosed 14 cases, 2 have been treated by Amphotericin B. The first was a chronic case with acute episodes which were less severe when Amphotericin B was used. This case has been alive for 20 months. The second case was an acute one with clinical and spinal fluid normalization and has a follow-up of 11 months.

In both cases Amphotericin was used intravenously $(50 \mathrm{mg}$ in $500 \mathrm{ml}$ of a solution of glycose at $5 \%$ ) in the first case 4 series and in the second case 2 series. 
There were no complications. The authors consider that the use of Amphotericin $\mathrm{B}$ has given those cases a better prognosis.

\section{REFERENCIAS}

1. ABBOT, K. H.; CUTLER, O. I. - Chronic coccidioidal meningitis. Arch. Pathol., 21:320-330, 1936. 2. AlmeIDA, F.; LACAZ, C. S. - Micose pelo Cryptococcus neoformans (10 caso observado em São Paulo). An. Paul. Med., 42:385-394, 1941. 3. ALMEIDA, F.; LACAZ, C. S.; MONTEIRO SALES, F. J. - Blastomicose do tipo Busse-Buschke (granulomatose criptocócica, torulose). An. Fac. Med. Univ. São Paulo, 20:115-139, 1944. 4. AMORIM, M. F.; PASQUALLUCCI, M. E. A. - Natureza das lesões do sistema nervoso central na torulose. Rev. Latino-Amer. de Anatomia Patológica, 7:41-50, 1958. 5. APPELBAUM, E.; SHTOKALKO, S. - Cryptococcus meningitis arrested with Amphotericin B. An. Int. Med., 47:346-351, 1957. 6. BEESON, P. B. - Cryptococcic meningitis of nearly sixteen years duration. Arch. Int. Med., 89:797-801, 1952. 7. CALDWELL, D. C.; RAPHAEL, S. S. - A case of cryptococcal meningitis. J. Clin. Path., 8:32-37, 1955. 8. CARTON, C. A. - Neurosurgical aspects of cryptococcosis. J. Neurosurg., 8:143-156, 1951. 9. CARTON, C. A. Treatment of central nervous system cryptococcosis: review and report of four cases treated with Actidione. An. Int. Med., 37:123-154, 1952. 10. CARTON, C. A.; LIEBIG, C. S. - Treatment of central nervous system cryptococcosis. Arch. Int. Med., 91:773-783, 1953. 11. CHAMPION DE CRESPIGNY, C. T. Torula infection of the central nervous system. Med. J. Australia, 2:605-615, 1944. 12. CLAUSELL, D. T. - Infecção primitiva do sistema nervoso central por Torulopsis neoformans (Torula histolytica): relato de um caso. An. Fac. Med. de Pôrto Alegre, 9:71-77, 1949. 13. COHEN, M. - Binocular papilledema in case of Torulosis associated with Hodgkin's disease. Arch. Ophth., 32:477-479, 1944. 14. COLLINS, V. P. - Bone involvement in cryptococcosis (Torulosis). Am. J. Roentgenol., 63:102-104, 1950. 15. CONANT, N. F.; MARTIN, D. S.; SMITH, D. T.; BAKER, R. D.; CALLAWAY, J. L. - Manual of Clinical Micology. W. B. Saunders Co., Filadélfia-Londres, 1944. 16. CORTEZ, J. M. - Criptoccocose pulmonar. An. Paul. Med. Cir., 58:315-329, 1949. 17. COX, L. B.; TOlHURST, J. C. - Cit. por Mosberg e col. ${ }^{11}$. 18. CRUTCHER, J. - Fungizone for infusion. Squibb Technical Leaflet, 1958. 19. DANIEL, P. M.; SCHILLER, F.; VOLLUM, R. L. - Torulosis of the central nervous system. Lancet, 256:53-56, 1949. 20. DELABARRE, J. - Fungizone for infusion. Squibb Technical Leaflet, 1958. 21. DIAMOND. H. - Fungizone for infusion. Squibb Technical Leaflet, 1958. 22. DICKIE, H. - Fungizone for Infusion. Squibb Technical Leaflet, 1958. 23. DROUET, E.; SEGRETAIN, G. - The action of hyaluronidase on the capsule of Torula histolytica. Compt. Rendu Acad. Sc., 228:424-425, 1949. 24. DUARTE, E. - Criptococose generalizada: apresentação de um caso com autópsia completa. Hospital, 43:345-361, 1953. 25. ECHOLS, D.; GARCIA, J. - Circular do Lab. Up John, 1949. 26. FIALHO, A. - Sôbre um caso de micose pulmonar e meníngea produzida pelo Cryptococcus neoformans. Brasil Méd., 66:201-202, 1952. 27. FISCHER, A. M. - The clinical picture associated with infections due to Cryptococcus neoformans. Bull. John Hopkins Hosp., 86:383-414, 1950. 28. FITZPATRICK, M. Fungizone for infusion. Squibb Technical Leaflet, 1958. 29. FITZPATRICK, M. J.; RUBIN, H.; POSER, C. M. - The treatment of cryptococcal meningitis with Amphotericin B; a new fupgicidal agent. An. Int. Med., 49:249-259, 1958. 30. FLINN, L. B.; HOOKER, J. W.; SCOTT, E. G. - Torula histolytica (Cryptococcus hominis) infection; case refractory to sulfonamides (Sulfathiazole and Sulfadiazine). Delaware State M. J., 18:141-147, 1946. 31. GEEVER, E. F.; CARTER, H. R.; NUEBUERGER, K. T.; SCHMIDT, E. A. - Roentgenologic and pathologic aspects of pulmonary tumors probably alveolar in origin, with report one of them complicated by torulosis of the central nervous system. Radiology, 44:319-327, 1945. 32. GENDEL, B. B.; ENDE, M.; NORMAN, S. L. - Cryptococcosis: A review with special reference to apparent association with Hodgkin's disease. Am. J. Med., 9:343-355, 1950. 33. 
GLobUS, J. H.; GANG, K. M.; BERGMAN, P. S. - Cit. por Wilson e Duryea ${ }^{99}$. 34. GOLD, W.; STOUT, H. A.; PAGANO, F. J.; DONOVICK, R. - Amphotericin A and B: antifungal antibiotics produced by a streptomycete in vitro-studies. Antibiotics Ann., 579-581, 1955-1956. 35. GOLDBERG, F. A. - Torula meningitis. Memphis M. J., 23:129-130, 1948. 36. GOLDBERG, L. H. - Infection of central nervous system: case with necropsy findings. J Lab. a. Clin. Med., 26:299-301, 1940.37. GREENFIELD, J. G.; MARTIN, J. P. MOORE, M. T. - Meningo-encephalitis due to Cryptococcus meningitidis (Torula histolytica: with report of a case. Lancet, 235:1154-1157, 1938. 38. GREENING, R. R.; MENVILLE, L. J. - Roentgen findings in torulosis: report of four cases. Radiology, 48:381-388, 1947. 39. GREENWALD, L. M. - Cit. por Carton ?. 40. HAGEN, W. S. - Torula histolytica meningoencephalites; report of a case with spinal fluid studies and autopsy report. The Military Surgeon, 94:29-34, 1944. 41. HALDE, C.; Mc NALL, E. G.; NEW COMER, V. D.; STERNBERG, T. H. - Properdin levels in mice and man with coccidioidomycosis during soluble Amphotericin B administration. Antibiotics Annual, 598-601, 1957-1958. 42. HAMILTON, L. C.; THOMPSON, P. E. - Treatment of cryptococcic meningitis with penicillin. Am. J. Dis. Children, 72:334-342, 1946. 43. HASPEL, R.; BAKER, J.; MOORE Jr., M. B. - Disseminated Cryptococcus neoformans. New Orleans M. S. J., 101:573-575, 1949. 44. HASSIN, G. B. - Torulosis of the central nervous system. J. Neuropathol. a. Exper. Neurol., 6:44-60, 1947. 45. HEMPHILL, J. J.; HERMAN, Y. F.; MAE YOUNG, V. - Comparative antifungal activity of Nystatin and Amphotericin B in tissue culture for virus propagation. Antibiotics Annual, 961-966, 1957-1958. 46. HIGGINS, J. M. - Circular do Lab. Up John, 1949. 47. HILLS, J. - Fungizone for infusion. Squibb Technical Leaflet, 1958. 48. HOBBY, G. L.; REGNA, P. P.; DOUGHERTY, N.; STIEG, W. E. - The antifungal activity of antibiotic XG. J. Clin. Investigation, 28:927-933, 1949. 49. HOLDEN, M.; SEEGAL, B. C.; BAER, H. - Range of antibiotic activity of protoanemonin. Proc. Soc. Exper. Biol. a. Med., 66:54-60, 1947. 50. HOLMES, S. J.; HAWKS, G. H. Torulosis of the central nervous system. Canad. M. A. J., 68:143-146, 1953.51. HOLT, G. - Fungizone for infusion. Squibb Technical Leaflet, 1958. 52. JOHNSON, W. - Fungizone for infusion. Squibb Technical Leaflet, 1958. 53. JONES, S. H.; KLINDK Jr., G. - Torula histolytica (Cryptococcus hominis) meningitis: report and therapeutic experiments. An. Int. Med., 22:736-745, 1945. 54. KLIGMAN, A. M.; WEIDMAN, F. D. - Experimental studies on treatment of human torulosis. Arch. Dermatol. a. Syphil., 60:726-741, 1949. 55. KUHN, L. R. - Growth and viability of Cryptococcus hominis at mouse and rabbit body temperatures. Proc. Soc. Exper. Biol. a. Med., 41:573-574, 1939. 56. KWHN, L. R. - Effect of elevated body temperatures on cryptococcosis in mice. Proc. Soc. Exper. Biol. a. Med., 71: 341-343, 1949. 57. LACAZ, C. S.; LAMARTINE DE ASSIS, J.; BITTENCOURT, J. M. T. - Micoses do sistema nervoso. Arq. Neuro-Psiquiat., 5:1-52, 1947 . 58. LEHAN, P. H.; YATES, I. L.; BRASHER, C. A.; LARSH, H. W.; FURCOLOW, M. L. Experiences with the therapy of sixty cases of deep mycotic infections. Dis. Chest, 32:597, 1957. 59. LEVIN, E. A. - Torula infection of the central nervous system. Arch. Int. Med., 59:667-684, 1937. 60. LEVY, I. - Circular do Lab. Up John, 1949. 61. LEWIN, W.; ROUX, P. - Cit. por Mosberg e Arnold ${ }^{71}$. 62. LITTMAN, M.; GRAHAM, F. - Fungizone for infusion. Squibb Technical Leaflet, 1958. 63. LITTMAN, M. L.; ZIMMERMAN, L. E. - Cit. por Rubin e Furcolow ${ }^{82}$. 64. LYNCH Jr., F. B.; ROSE, E. - Torula meningitis. Ann. Clin. Med., 4:456, 1926.65. MARSHALL, M.; TEED, R. W. - Torula histolytica meningoencephalitis. Recovery following bilateral mastoidectomy and sulfonamide therapy; preliminary report. J.A.M.A., 120: 527-530, 1942. 66. MC NALL, E. G.; HALDE, C.; NEWCOMER, V. D.; THOMAS, H. STERNBERG - A biological essay for the determination of Amphoreticin A and B in biological fluids. Antibiotics Annual, 131-136, 1957-1958. 67. MAGRUDER, R. G. - A report of three cases of Torula infection of the central nervous system. J. Lab. Clin. Med., 24:495-499, 1939. 68. MEZEY, C. M.; FOWLER, R. — Cerebrospinal cryptococcosis. J.A.M.A., 132:632-634, 1946. 69. MILLER, J. M.; SCHOENBACH, E. B.; LONG, P. H.; SHUTTLEWORTH, J. S.; SNIDER, G. E. - Treatment of infections due to Cryptococcus neoformans with Stilbamidine. Antibiotics and Chemotherapy, 
11:444-446, 1952. 70. MOSBERG, W. H.; ALVAREZ DE CHOUdENS, J. A. - Torulosis of the central nervous system: effect of changes in $\mathrm{pH}$ and temperature on growth of the causal organism. Lancet, 260:1269-1272, 1951. 71. MOSBERG Jr., W. H.; ARNOLD, J. G. - Torulosis of the central nervous system: review of literature and report of five cases. An. Int. Med., 32:1153-1183, 1950. 72. NEUHAUSER, E. B. D.; TULKER, A. - The roentgen changes produced by diffuse torulosis in the newborn. Am. J. Roentgenol., 59:805-815, 1948. 73. NICHOLS, I. C. - Torula meningoencephalitis: report of a case. Rhode Island M. J., 24:221-223, 1941. 74. PADBERG, F.; MARTIN, J. - Torulosis of the brain: a case report. J. Neurosurg., 9: 307-309, 1952. 75. PERONCINI, J.; BENCE, A. E.; BACCAREZZA, O. A.; AGUERO, J. G. - Torulosis bronquial y meningea. Medicina (Buenos Aires), 9:363-370, 1949. 76. REEVES, D. L.; BUTT, E. M.; HAMMACK, R. W. - Torula infection of the lungs and cerebral nervous system. Arch. Int. Med., 68:57-79, 1941. 77. REILLY, E. B. 2 ARTMAN, E. L. - Cryptococcosis: report of a case and experimental studies. Arch. Int. Med., 81:1-8, 1948. 78. REIS, J. B.; BEI, A. - O liqüido cefalorraqueano no diagnóstico da criptococcose do sistema nervoso. Arq. Neuro-Psiquiat., 14:201212, 1956. 79. ROANTREE, W. B.; DUNKERLEY, G. E. - Meningo-encephalitis due to Cryptococcus neoformans. Lancet, 262:1274-1278, 1952. 80. ROBERTSON Jr., H. C.; MOSELEY, V. - Cryptococcus meningitis: report of case with survival after 14 months. Ann. Int. Med., 36:1538-1550, 1952. 81. ROBINSON, H. J.; SMITH, D. G.; GRAESSLE, O. E. - Chemotherapeutic properties of Streptomycin. Proc. Soc. Exper. Biol. a. Med., 57:226-231, 1944. 82. RUBIN, H.; FURCOLOW, M. L. - Promissing results in cryptococcal meningitis. Neurology, 8:590-595, 1958. 83. SAMPSON, B. F.; FARREN, J. E. - Another case of Torula meningitis. South African M. J., 16:245, 1942. 84. SEEGAL, B. C. - Cit. por Carton ${ }^{\circ}$. 85. SHAPIRO, L. L.; NEAL, J. B. Torula meningitis. Arch. Neurol. a. Psychiat., 13:147-190, 1925. 86. SHEPPE, W. M. - Torula infection in man. Am. J. Medical Sci., 167:91-107, 1924 87. SILVA, N. N. - Criptococose cutânea. Hospital (Rio de Janeiro), 44:375-381, 1953. 88. STEINBERG, A. B.; JAMBOR, W. P.; SUYDAN, L. O. - Amphotericin A and B two new antifungal antibiotics possessing high activity against deep-seated and superficial mycoses. Antibiotics Annual, 574-575, 1955-1956. 89. STONE, W. Y.; STURDIVANT, B. F. - Meningo-encephalitis due to Torula histolytica. Arch. Int. Med., 44:560575, 1929. 90. SWANSON, H. S.; SMITH, W. A. - Torular granuloma simulating cerebral tumor. Arch. Neurol. a. Psychiat., 51:426-431, 1944. 91. SWARTZ, M. Fungizone for infusion. Squibb Technical Leaflet, 1958. 92. TINNEY, W. S.; SCHMIDT, H. W. - Torula infection. M. Clin. North. Am., 28:950-956, 1944. 93. TOLOSA, A.; SPINA FRANÇ, A.; LACAZ, C. S. - Criptococose do sistema nervoso central: registro de um caso. Arq. Neuro-Psiquiat., 14:171-178, 1956. 94. UTTERBACK, R. - Fungizone for infusion. Squibb Technical Leaflet, 1958. 95. UTZ, J. P.; LOURIA, D. B.; FEDER, N.; EMMONS, C. W.; Mc CULlOUGH, N. B. - A report of clinical study on the use of the Amphotericin $B$ in patients with systemic fungal diseases. Antibiotics Annual, 65:70, 1957-1958. 96. VOYLES, C. G.; BECK, E. M. - Systemic infections due to Torula histolytica: report of four cases and review of the literature. Arch. Int. Med., 77:504-515, 1946. 97. WARVI, W. N.; RAWSON, R. W. - Torula meningitis. Arch. Int. Med., 69:90-98, 1942. 98. WATTS, J. W. - Torula infection: a review and report of two cases. Am. J. Pathol., 8: 167-192, 1932. 99. WILSON, H. M.; DURYEA, A. W. - Cryptococcus meningitis (torulosis) treated with a new antibiotic Actidione. Arch. Neurol. a. Psychiat., 66: 470-480, 1951. 100. WILSON, J. W. - Cryptococosis. J. Chronic Dis., 5:445-459, 1957. 101. LONGO, P. W.; DINIZ, H. B.; PIMENTA, A. M.; MANGABEIRA ALBERNAZ, P. - Simpósio sôbre criptococose no Departamento de Neuro-Psiquiatria da Associação Paulista de Medicina em 13 de setembro de 1955. Rev. Paulista Med., 48:196-198, 1956. 102. BENfCIO, A. - Comunicação pessoal. 